\title{
On the Validity of Ward Identities
}

\author{
P. STICHEL \\ II. Institut für Theoretische Physik der Universität Hamburg \\ und \\ Deutsches Elektronen-Synchrotron DESY, Hamburg
}

Received April 28, 1970

\begin{abstract}
Ward identities for matrix elements of covariant two-point time-ordered operators in the presence of an arbitrary number of subtractions are investigated. Neither the existence of naive $T$-products nor the existence of equal-time commutators between current densities will be assumed. It is shown by means of the Jost-Lehmann-Dyson representation that $T^{*}$-products can always be defined such that normal Ward identities with respect to one current are valid. The simultaneous validity of normal Ward identities with respect to two currents requires a relation between equal-time charge-current commutators.

Our results show that the usual realization of current algebra in the form of Ward identities is possible even if subtractions are necessary. Some examples are discussed in detail.
\end{abstract}

\section{Introduction}

Ward identities (WI's) are of great importance for the derivation of low energy theorems [1]. In particular they are the basis for the application of current algebra in the form of the hard-pion methods [2]. Therefore, it is of great interest to investigate in detail the validity of such WI's.

In this paper we attack the problem of the validity of normal WI's for matrix elements of time-ordered products in the framework of general quantum field theory.

We exclusively consider the case of $T$-products of two field operators where at least one will be a current. Then we speak about a normal WI if a relation of the following kind is valid

with

$$
\begin{gathered}
\partial_{x}^{\mu}\left\langle\Psi_{2}\left|T\left(j_{\mu}(x) A_{\gamma}(y)\right)\right| \Psi_{1}\right\rangle=\left\langle\Psi_{2}\left|T\left(\partial^{\mu} j_{\mu}(x) A_{\gamma}(y)\right)\right| \Psi_{1}\right\rangle \\
+\delta^{4}(x-y)\left\langle\Psi_{2}\left|\left[Q_{(j)}\left(x_{0}\right), A_{\gamma}(y)\right]\right| \Psi_{1}\right\rangle
\end{gathered}
$$

$$
Q_{(j)}\left(x_{0}\right) \equiv \int d^{3} x j_{0}(x)
$$


for any local field $A_{\gamma}$ and arbitrary quasilocal states $\Psi_{1}$ and $\Psi_{2}$. Historically, (I) has been derived first by using the naive definition of the $T$-product ${ }^{1}$ and canonical equal-time commutators (ETC) between $j_{0}$ and $A_{\gamma}[3]$.

This derivation has several shortcomings:

a) The naive $T$-product may not exist. This happens in perturbation theory, e.g. if a Feynman amplitude diverges and, therefore, needs subtractions.

b) Even if the naive $T$-product exists it might be non covariant [1].

c) In case of non-conserved currents the ETC between a "charge" and the local field $A_{\gamma}$ may not exist. A perturbation theoretical example for such a situation has been given recently by the present author [5].

d) Even if the ETC between $Q_{(j)}$ and $A_{\gamma}$ exists the ETC between $j_{0}$ and $A_{\gamma}$ might deviate from its canonical value (appearance of gradient terms - so-called "Schwinger terms" - which also may be infinite $[5,6]$ ).

Let us now briefly discuss what people have been done in the last couple of years in order to overcome these difficulties.

First one trivial remark: As we ask for the validity of a normal WI we must require that the ETC between a charge and the field $A_{\gamma}$ exists. In most of the recent nonperturbation theoretic work on WI's it has been assumed that

$\alpha)$ the naive $T$-product exists,

$\beta$ ) ETC's between the local operators $j_{\mu}$ and $A_{\gamma}$ exist.

Then the question may be asked whether the divergence of the seagull term - which has to be added to the naive $T$-product in order to get a covariant $T$-product - and the Schwinger term in the ETC cancel each other. First this problem has been examined in the framework of canonical Lagrangian field theories resulting in a positive answer [7]. But the derivations given by these authors can be considered as formal only because the difficulties met for the definition of products of field operators at the same space-time point have been ignored completely.

More recent work did not refer to Lagrangian field theory but only assumptions $\alpha$ ) and $\beta$ ) have been made with the restriction that at most first order Schwinger terms appear in current-current ETC's. Gross and Jackiw [8] assumed in addition that for the case of two-currents $\left(A_{\gamma}=g_{v}\right)$ the naive $T$-product $T\left(\partial^{\mu} j_{\mu}(x) g_{v}(y)\right)$ is covariant. Their result is [8]:

${ }^{1}$ We speak about naive $T$ - or $R$-products if the multiplication of a distribution $f(x)$ with the step function $\Theta\left(x_{0}\right)$ is defined by means of an unsubtracted Hilbert transform in momentum space, i.e. $\widetilde{\Theta f}(q)=\frac{1}{2 \pi i} \int d q_{0}^{\prime} \frac{\tilde{f}\left(q_{0}^{\prime}, \boldsymbol{q}\right)}{q_{0}^{\prime}-q_{0}-i \varepsilon}$. 
1) The WI with respect to one current (called "single WI" in the following) can always be satisfied by means of a suitable choice of the seagull.

2) Simultaneous WI's for both currents (called "double WI" in the following) require a special form of the Schwinger term in the $\left[g_{0}, j_{0}\right]-$ ETC.

Similar work restricted to conserved currents but allowing for an arbitrary number of currents in the $T$-product has been done by Dashen and Lee [9]. As in their work no Schwinger term in the $\left[g_{0}, j_{0}\right]$-ETC has been allowed ${ }^{2}$ it is not surprising that all multiple WI's are satisfied after a suitable choice of the seagulls [9].

But very recently it has been recognized by Adler and Boulware [10] and other authors [11] that one may have irreparable deviations from normal WI's in perturbation theory if the corresponding Feyman amplitude is divergent, i.e. if the naive $T$-product does not exist.

It is, therefore, necessary to examine WI's in a general framework without assuming the existence of the naive $T$-product. Some work along that line has been done by Wilson [12] restricted to three-point functions and allowing for at most two subtractions.

It is the aim of the present paper to generalize the work of Wilson [12]. Therefore, we neither do assume the existence of naive $T$-products nor the existence of ETC's of current densities and also allow for an arbitrary number of subtractions in the definition of the $T^{*}$-product. For technical reasons (use of the Jost-Lehmann-Dyson representation) we don't work with the $T^{*}$-product but with the closely related retarded $(R)$-product defined covariantly ${ }^{3}$.

Unfortunately, due to the use of the Jost-Lehmann-Dyson representation we are restricted to the consideration of matrix-elements of twopoint retarded operators.

Single WI's and double WI's will be examined in Chapters 2 and 3 respectively. Chapter 4 is devoted to some applications of our general results to two-, three-, and four-point functions.

\section{Single Ward Identities}

In this chapter we examine the question whether the usual form of the Ward identity with respect to a single current $j_{\mu}(x)$ can be obtained by means of a suitable redefinition of the covariant $R$-product $R\left(j_{\mu}(x) A_{\gamma}(y)\right)$.

\footnotetext{
${ }^{2}$ Exactly this happens in the Lagrangian framework [7].

${ }^{3}$ It is immediately clear that WI's for $T^{*}$-products and covariant $R$-products respectively have the same form.
} 
2.1. General Form of $\partial_{x}^{\mu} R\left(j_{\mu}(x) A_{\gamma}(y)\right)$. We start with some definitions and notations.

Commutator Matrix Elements.

$$
F_{j_{\mu} A_{\gamma}}(x) \equiv\left\langle\Psi_{2}\left|\left[j_{\mu}(x / 2), A_{\gamma}(-x / 2)\right]\right| \Psi_{1}\right\rangle
$$

where $A_{\gamma}$ is an arbitrary local field operator ( $\gamma$ describes the tensor character of $A$ ) and where the states $\left|\Psi_{i}\right\rangle$ are arbitrary particle states with sharp momentum $p_{i}$.

$$
F_{j A_{\gamma}}(x) \equiv\left\langle\Psi_{2}\left|\left[\partial^{\mu} j_{\mu}(x / 2), A_{\gamma}(-x / 2)\right]\right| \Psi_{1}\right\rangle .
$$

Retarded Commutator Matrix Elements.

$$
F_{j_{\mu} A_{\gamma}}^{R}(x) \equiv \Theta\left(x_{0}\right) F_{j_{\mu} A_{\gamma}}(x) \quad \text { etc. }
$$

This is a formal definition of the retarded commutator. A precise definition in momentum space will be given below.

Fourier Transforms.

$$
\tilde{F}_{j_{\mu} A_{\gamma}}(q) \equiv \int d^{4} x e^{i q x} F_{j_{\mu} A_{\gamma}}(x) \text { etc. }
$$

According to (1), (2), and (4) we have then

with $\Delta \equiv p_{2}-p_{1}$.

$$
-i(q-\Delta / 2)^{\mu} \tilde{F}_{j_{\mu} A_{\nu}}(q)=\tilde{F}_{j A_{\nu}}(q)
$$

Since $j_{\mu}$ and $A_{\gamma}$ are supposed to be local fields, our commutator matrix elements satisfy the Jost-Lehmann-Dyson (JLD)-representation $[13,14]$. In particular we have for $\tilde{F}_{j A_{\gamma}}(q)$

$$
\tilde{F}_{j A_{\gamma}}(q)=\int d^{4} u \int d s \varepsilon\left(q_{0}-u_{0}\right) \delta\left((q-u)^{2}-s\right) \varphi(u, s)
$$

with the usual support of $\varphi[14]$.

Now we need the JLD-representation for $\tilde{F}_{j_{\mu} A_{\gamma}}(q)$ in such a form that (5) and (6) are satisfied explicitly. This problem has been solved by the Völkels [15]:

$$
\begin{aligned}
\tilde{F}_{j_{\mu} A_{\gamma}}(q)= & i \int d^{4} u \int d s \varepsilon\left(q_{0}-u_{0}\right) \delta\left((q-u)^{2}-s\right) \\
& \cdot\left[P \frac{(q+\Delta / 2-2 u)_{\mu}}{s-(u-\Delta / 2)^{2}}\left((q-\Delta / 2)_{v} W^{v}(u, s)+\varphi(u, s)\right)\right. \\
& \left.+(q+\Delta / 2-2 u)_{\mu} \delta\left(s-(u-\Delta / 2)^{2}\right) E(u, s)-W_{\mu}(u, s)\right] .
\end{aligned}
$$


We may define covariant retarded commutator matrix elements by means of $N$-fold subtracted JLD-representations

$$
\begin{aligned}
\tilde{F}_{j_{\mu} A_{\nu}}^{R}(q)= & -\frac{1}{2 \pi} \int d^{4} u \int d s \frac{\left((q-u)^{2}+a^{2}\right)^{N}}{\left(s+a^{2}\right)^{N}\left((q-u+i \varepsilon)^{2}-s\right)} \\
& \cdot\left[P \frac{(q+\Delta / 2-2 u)_{\mu}}{s-(u-\Delta / 2)^{2}}\left((q-\Delta / 2)^{v} W_{v}(u, s)+\varphi(u, s)\right)\right. \\
& \left.-W_{\mu}(u, s)\right]-\frac{1}{2 \pi} \int d^{4} u \int d s \frac{(q+\Delta / 2-2 u)_{\mu}}{(q-u+i \varepsilon)^{2}-s} \\
& \cdot \delta\left(s-(u-\Delta / 2)^{2}\right) E(u, s)+P_{(2 N) \mu}^{(1)}(q)
\end{aligned}
$$

where we denote by $P_{(\kappa)}(q)$ a polynomial in $q$ of order $\kappa$. The number $N$ in (8) should be understood to be the smallest positive integer such that each term exists separately. From this follows that $\tilde{F}_{j A_{\gamma}}^{R}$ obeys an JLDrepresentation with at most $N+1$ subtractions.

$\tilde{F}_{j A_{\gamma}}^{R}(q)=\frac{i}{2 \pi} \int d^{4} u \int d s \frac{\left((q-u)^{2}+a^{2}\right)^{N+1} \varphi(u, s)}{\left(s+a^{2}\right)^{N+1}\left((q-u+i \varepsilon)^{2}-s\right)}+P_{(2 N)}^{(2)}(q)$.

By means of an elementary calculation we may now compute

and obtain

$$
-i(q-\Delta / 2)^{\mu} \tilde{F}_{j_{\mu} A_{\nu}}^{R}(q)
$$

$$
\begin{aligned}
-i(q-\Delta / 2)^{\mu} \tilde{F}_{j_{\mu} A_{\gamma}}^{R}(q)= & \tilde{F}_{j A_{\gamma}}^{R}(q)+\frac{i}{2 \pi} \int d^{4} u \int d s \delta\left(s-(u-\Delta / 2)^{2}\right) \\
& \cdot E(u, s)+P_{(2 N+1)}^{(3)}(q)
\end{aligned}
$$

with

$$
\begin{aligned}
& P_{(2 N+1)}^{(3)}(q)=-i(q-\Delta / 2)^{\mu}\left[P_{(2 N)_{\mu}}^{(1)}(q)-\frac{1}{2 \pi} \int d^{4} u \int d s\right. \\
& \left.\cdot P \frac{\left((q-u)^{2}+a^{2}\right)^{N} W_{\mu}(u, s)}{\left(s+a^{2}\right)^{N}\left(s-(u-\Delta / 2)^{2}\right)}\right]+\frac{i}{2 \pi} \int d^{4} u \int d s P \frac{\left((q-u)^{2}+a^{2}\right)^{N}}{\left(s+a^{2}\right)^{N}} \\
& \cdot \frac{(u-\Delta / 2)^{2}+a^{2}}{s-(u-\Delta / 2)^{2}} \varphi(u, s)-P_{(2 N)}^{(2)}(q) .
\end{aligned}
$$

The sum of the last two terms in (10)' can be developed into a power series in $q-\Delta / 2$. Therefore, we have due to ( 9 )

$$
\begin{aligned}
P_{(2 N+1)}^{(3)}(q)= & -i(q-\Delta / 2)^{\mu}\left[P_{(2 N)_{\mu}}^{(1)}(q)+P_{(2 N)_{\mu}}^{(4)}(q)\right] \\
& -\tilde{F}_{j A_{\gamma}}^{P}(\Delta / 2)
\end{aligned}
$$


where the upper index $P$ in the last term means that in (9) the principal value integral has to be taken.

The normal Ward identity requires the $q$-independence of $P_{(2 N+1)}^{(3)}(q)$ in (10). According to (11) this is the case if we choose e.g.

$$
P_{(2 N)_{\mu}}^{(1)}(q)+P_{(2 N)_{\mu}}^{(4)}(q)=0 .
$$

In order to decide whether (12) is sufficient for getting a normal WI we have to distinguish several cases. This will be done in the following sections.

2.2. Conserved Current. The conservation law $\partial^{\mu} j_{\mu}=0$ is equivalent to $\tilde{F}_{j A_{\gamma}}(q) \equiv 0$. This does not imply $\varphi(u, s) \equiv 0$ since due to the nonuniqueness of the Dyson representation there exist non-trivial distributions $\varphi$ such that

holds.

$$
\int d^{4} u \int d s \varepsilon\left(q_{0}-u_{0}\right) \delta\left((q-u)^{2}-s\right) \varphi(u, s) \equiv 0
$$

The requirement $\tilde{F}_{j A_{\gamma}}^{R}(q) \equiv 0$ we, therefore, fulfill by means of a suitable choice of the polynomial $P_{(2 N)}^{(2)}(q)$ in (9). In any case, the redefinition of our retarded product due to (12) means in the conserved case that $\varphi$ has been eliminated in (8). We obtain for our redefined $\tilde{F}_{j_{\mu} A_{\gamma}}^{R}(q)(9)$ according to Eqs. $(10)^{\prime}-(12)$

$\tilde{F}_{j_{\mu} A_{\gamma}}^{R}(q)=-\frac{1}{2 \pi} \int d^{4} u \int d s \frac{(q+\Delta / 2-2 u)_{\mu}}{(q-u+i \varepsilon)^{2}-s} \delta\left(s-(u-\Delta / 2)^{2}\right) E(u, s)$

$-\frac{1}{2 \pi} \int d^{4} u \int d s \frac{\left((q-u)^{2}+a^{2}\right)^{N}}{\left(s+a^{2}\right)^{N}\left((q-u+i \varepsilon)^{2}-s\right)}\left[P \frac{(q+\Delta / 2-2 u)_{\mu}}{s-(u-\Delta / 2)^{2}}(q-\Delta / 2)_{v} W^{v}\right.$

$\left.-P W_{\mu} \frac{(q-u)^{2}-(u-\Delta / 2)^{2}}{s-(u-\Delta / 2)^{2}}\right]$.

We note that

a) the only arbitrariness in (14) is due to the choice of the subtraction point $a^{2}$,

b) instead of $N$ subtractions we only need $N-1$.

The following statement shows that our redefined retarded commutator satisfies the normal WI.

Statement. In the case of a conserved current we have

$\frac{i}{2 \pi} \int d^{4} u \int d s \delta\left(s-(u-\Delta / 2)^{2}\right) E(u, s)=\left\langle\Psi_{2}\left|\left[Q_{(j)}\left(x_{0}\right), A_{\gamma}(0)\right]\right| \Psi_{1}\right\rangle$. 
Proof. By means of translational invariance and the definition of the Fourier transform we get

$$
\left\langle\Psi_{2}\left|\left[Q_{(j)}\left(x_{0}\right), A_{\gamma}(0)\right]\right| \Psi_{1}\right\rangle=\frac{1}{2 \pi} \int d q_{0} \tilde{F}_{j_{0} A_{\gamma}}\left(q_{0}, \Delta / 2\right) .
$$

On the other hand we obtain by means of (7) and (13)

$$
\int d q_{0} \tilde{F}_{j_{0} A_{\gamma}}\left(q_{0}, \Delta / 2\right)=i \int d^{4} u \int d s \delta\left(s-(u-\Delta / 2)^{2}\right) E(u, s)
$$

because

a) the $W$-part of (7) does not contribute to $\tilde{F}_{j_{0} A_{\gamma}}\left(q_{0}, \Delta / 2\right)$,

b) the $\varphi$-contribution to $\tilde{F}_{j_{0} A_{\gamma}}\left(q_{0}, \Delta / 2\right)$ may be rewritten as

$$
i P \frac{1}{q_{0}-\Delta_{0} / 2} \int d^{4} u \int d s \delta\left((q-u)^{2}-s\right)_{\boldsymbol{q}=\boldsymbol{\Delta} / 2} \varepsilon\left(q_{0}-u_{0}\right) \varphi(u, s)
$$

which vanishes due to (13).

2.3. Non-Conserved PCAC-Current. We define PCAC by means of the requirement that $\tilde{F}_{j A_{\gamma}}^{R}(q)$ defined by (9) does not need any subtraction ${ }^{4}$.

Then our redefined retarded commutator satisfies the normal WI, because according to (9) and (7) we see immediately that

$$
\begin{gathered}
\frac{i}{2 \pi} \int d^{4} u \int d s \delta\left(s-(u-\Delta / 2)^{2}\right) E(u, s)-\tilde{F}_{j A_{\gamma}}^{p}(\Delta / 2) \\
=\left\langle\Psi_{2}\left|\left[Q_{(j)}(0), A_{\gamma}(0)\right]\right| \Psi_{1}\right\rangle .
\end{gathered}
$$

2.4. Non-PCAC Current. A non-conserved current will be called a nonPCAC current if the representation for $\tilde{F}_{j A_{\gamma}}^{R}(q)$ in (9) requires at least one subtraction. Then we have in any case a free constant in the definition of $\tilde{F}_{j A_{\gamma}}^{R}(q)$, i.e. $\tilde{F}_{j A_{\gamma}}^{P}(\Delta / 2)$ is arbitrary and, therefore,

$$
-i(q-\Delta / 2)^{\mu} \tilde{F}_{j_{\mu} A_{\gamma}}^{R}(q)-\tilde{F}_{j A_{\gamma}}^{R}(q)
$$

can take any desired value. In general, the ETC between $Q_{(j)}$ and $A_{\gamma}$ will not exist in this case, but we can always choose our free constant such that we have a "naive-normal" WI where the r.h.s. is given by means of the "naive-canonical" ETC.

\section{Double Ward Identities}

In this chapter we put the field $A_{\gamma}(y)$ equal to another current $g_{v}(y)$ and ask whether the WI's with respect to both currents $j_{\mu}$ and $g_{v}$ can be satisfied simultaneously.

${ }^{4}$ This definition has to be changed if $A_{\gamma}$ is a current too. Compare for this case Section 3.2 below.

20 Commun. math. Phys., Vol. 18 
In order to simplify the notation we use the following abbreviations:

$$
\begin{aligned}
F_{\mu \nu} & \equiv F_{j_{\mu} g_{v}}, \quad F_{j_{\mu}} \equiv F_{j_{\mu} g} \quad \text { etc. }, \\
q_{ \pm} & \equiv q \pm \Delta / 2 .
\end{aligned}
$$

3.1. Conserved Currents. According to Chapter 2 we may always choose $\tilde{F}_{\mu v}^{R}(q)$ in such a way that the WI with respect to $j_{\mu}$ is satisfied, i.e. we have

$$
-i q_{-}^{\mu} \tilde{F}_{\mu \nu}^{R}(q)=\left\langle\Psi_{2}\left|\left[Q_{(j)}(0), g_{v}(0)\right]\right| \Psi_{1}\right\rangle .
$$

Furthermore, the WI with respect to $g_{v}$ has the form

$$
\begin{aligned}
i q_{+}^{v} \tilde{F}_{\mu \nu}^{R}(q)= & -\left\langle\Psi_{2}\left|\left[j_{\mu}(0), Q_{(g)}\right]\right| \Psi_{1}\right\rangle \\
& -q_{+}^{v} P_{(2 N) \mu v}(q) .
\end{aligned}
$$

Now our problem may be stated as follows:

Find a polynomial $S_{\mu v}(q)$ such that

and

$$
q_{-}^{\mu} S_{\mu \nu}(q)=0
$$

$$
\begin{gathered}
i q_{+}^{v} S_{\mu v}(q)=q_{+}^{v} P_{\mu v}(q) \\
\left(P_{\mu \nu} \equiv P_{(2 N) \mu v}\right) .
\end{gathered}
$$

Then the redefined retarded commutator matrix element

$$
\tilde{F}_{\mu \nu}^{R^{\prime}}(q)=\tilde{F}_{\mu \nu}^{R}(q)+S_{\mu \nu}
$$

will satisfy both WI's simultaneously.

Statement 1. A polynomial $S_{\mu v}(q)$ satisfying (21) exists if and only if the condition

is fulfilled.

$$
q_{-}^{\mu} q_{+}^{v} P_{\mu \nu}(q)=0
$$

Proof. a) From (21) we see immediately that (23) is a necessary condition for the existence of $S_{\mu v}$.

b) The most general polynomial solution of (23) for $q_{+}^{v} P_{\mu \nu}$ is given by

$$
q_{+}^{v} P_{\mu \nu}=\varepsilon_{\mu v \varrho \sigma} q^{v} \Delta^{\varrho} A^{\sigma}+C_{[\mu, \varrho] v}(q) q_{-}^{\varrho} q_{+}^{v}
$$

because

$\alpha$ ) first we have from (23)

$$
q_{+}^{v} P_{\mu \nu}=C_{[\mu, \varrho]}(q) q_{-}^{\underline{e}}
$$

where, due to the 1.h.s. of (25)

$$
C_{[\mu, \varrho]}(-\Delta / 2) \Delta^{\varrho}=0,
$$


$\beta)$ define $C_{[\mu, e] v}(q)$ by means of

$$
C_{[\mu, \varrho]}(q)=C_{[\mu, \varrho]}(-\Delta / 2)+q_{+}^{v} C_{[\mu, \varrho] v}(q),
$$

$\gamma$ ) the solution of (26) has the form

$$
C_{[\mu, v]}(-\Delta / 2)=\varepsilon_{\mu \nu \varrho \sigma} \Delta^{\varrho} A^{\sigma} .
$$

$\delta$ ) insertion of (27) with (28) into (25) leads to (24).

c) with (24) we find the following particular solution of (21) for $S_{\mu \nu}$

$$
S_{\mu \nu}(q)=-\varepsilon_{\mu \nu \varrho \sigma} q_{-}^{\varrho} A^{\sigma}+q^{\varrho} C_{[\mu, \varrho] \nu}(q) .
$$

According to statement 2 the condition (23) may be reformulated in terms of physical quantities.

Statement 2. Condition (23) is equivalent to

$$
\left\langle\Psi_{2}\left|\left[Q_{(j)}, g_{\mu}(0)\right]\right| \Psi_{1}\right\rangle=\left\langle\Psi_{2}\left|\left[j_{\mu}(0), Q_{(g)}\right]\right| \Psi_{1}\right\rangle .
$$

Proof. a) Due to (19) and (20) Eq. (23) is equivalent to

$$
q_{+}^{\mu}\left\langle\Psi_{2}\left|\left[Q_{(j)}, g_{\mu}(0)\right]\right| \Psi_{1}\right\rangle=q_{-}^{\mu}\left\langle\Psi_{2}\left|\left[j_{\mu}(0), Q_{(g)}\right]\right| \Psi_{1}\right\rangle .
$$

b) From translational invariance and current conservation we have

$$
\begin{aligned}
O & =\Delta^{\mu}\left\langle\Psi_{2}\left|\left[Q_{(j)}, g_{\mu}(0)\right]\right| \Psi_{1}\right\rangle \\
& =\Delta^{\mu}\left\langle\Psi_{2}\left|\left[j_{\mu}(0), Q_{(g)}\right]\right| \Psi_{1}\right\rangle .
\end{aligned}
$$

c) Insertion of (32) into (31) together with the $q$-independence of the equal-time charge-current commutator matrix elements leads immediately to $(30)$.

3.2. PCAC-Currents. In this section we examine the case where one current $\left(j_{\mu}\right)$ is of the PCAC type and the other $\left(g_{v}\right)$ is either conserved or of the PCAC-type too.

Despite of the fact that $j_{\mu}$ is supposed to be a PCAC-current $\tilde{F}_{g_{v}}^{R}(q)$ can not obey an unsubtracted JLD-representation

$$
\tilde{F}_{g_{v}}^{R}(q)=\frac{i}{2 \pi} \int d^{4} u \int d s \frac{\varphi_{g_{\nu}}(u, s)}{(q-u+i \varepsilon)^{2}-s}
$$

because this corresponds to

leading to

$$
\lim _{s \rightarrow \infty} \varphi_{g_{v}}(u, s)=0
$$

$$
\int d q_{0} \tilde{F}_{g_{0}}\left(q_{0},-\Delta / 2\right)=0
$$


which is equivalent to

$$
\left\langle\Psi_{2}\left|\left[Q_{(g)}, \partial^{\mu} j_{\mu}(0)\right]\right| \Psi_{1}\right\rangle=0 .
$$

But in most cases of physical interest this ETC is different from zero (e.g. $g_{v}=$ isospin current, $j_{\mu}=$ axial vector current). One subtraction in the sense of Eq. (9) is taken into account automatically if we assume for $\tilde{F}_{g_{v}}^{R}(q)$ a representation of the form (8) with $N=0$

$$
\begin{aligned}
& \tilde{F}_{g_{v}}^{R}(q)=-\frac{1}{2 \pi} \int d^{4} u \int d s \frac{1}{(q-u+i \varepsilon)^{2}-s}\left[P \frac{(-q+\Delta / 2-2 u)_{v}}{s-(u+\Delta / 2)^{2}}\right. \\
& \cdot\left(-(q+\Delta / 2)_{\mu} W_{j}^{\mu}(u, s)+\varphi(u, s)\right)+(-q+\Delta / 2-2 u)_{v} \\
&\left.\cdot \delta\left(s-(u+\Delta / 2)^{2}\right) E_{j}(u, s)-W_{j v}(u, s)\right]+P_{(0), g_{v}}^{(2)} .
\end{aligned}
$$

Now we redefine $\tilde{F}_{\mu \nu}^{R}(q)$ such that

$$
-i(q-\Delta / 2)^{\mu} \tilde{F}_{\mu \nu}^{R}(q)-\tilde{F}_{g_{v}}^{R}(q)
$$

becomes $q$ independent i.e., due to Eqs. (10) and (11), equal to

$$
\frac{i}{2 \pi} \int d^{4} u \int d s \delta\left(s-(u-\Delta / 2)^{2}\right) E_{g_{v}}(u, s)-\tilde{F}_{g_{v}}^{P}(\Delta / 2) \stackrel{\text { def }}{\equiv} x_{g_{v}} .
$$

This corresponds to the situation discussed in section 2.4. But the arbitrariness of expression $(33 \mathrm{~b})$ can be lifted if we require that $\tilde{F}_{g_{v}}^{R}(q)$ satisfies a normal WI.

Statement 3. Suppose $\left\langle\Psi_{2}\left|R\left(\partial^{\mu} j_{\mu}, \partial^{v} g_{v}\right)\right| \Psi_{1}\right\rangle$ obeys an unsubtracted $J L D$-representation (PCAC-situation). Then $\tilde{F}_{\mu \nu}^{R}(q)$ satisfies a normal WI with respect to $j_{\mu}$ if and only if $\tilde{F}_{g_{v}}^{R}(q)$ satisfies a normal $W I$.

Proof. a) With the representation (33) for $\tilde{F}_{g_{v}}^{R}$ and the assumed unsubtracted JLD-representation for

$$
\left\langle\Psi_{2}\left|R\left(\partial^{\mu} j_{\mu}, \partial^{v} g_{v}\right)\right| \Psi_{1}\right\rangle
$$

we get according to the results of Chapter 2 a normal WI for $\tilde{F}_{g_{v}}^{R}$, i.e.

$$
i(q+\Delta / 2)^{v} \tilde{F}_{g_{\nu}}^{R}(q)=\tilde{F}^{R}(q)-\left\langle\Psi_{2}\left|\left[\partial^{\mu} j_{\mu}(0), Q_{(g)}(0)\right]\right| \Psi_{1}\right\rangle
$$

if we put

$$
P_{(0), g_{v}}^{(2)}=\frac{1}{2 \pi} \int d^{4} u \int d s \frac{W_{j v}(u, s)}{s-(u+\Delta / 2)^{2}} .
$$


b) With (33) and (35) we find by means of an explicit calculation that $\tilde{F}_{g_{0}}^{R}(q)$ is given by the naive retarded commutator matrix element

$$
\tilde{F}_{g_{0}}^{R}(q)=\frac{1}{2 \pi i} \int d q_{0}^{\prime} \frac{\tilde{F}_{g_{0}}\left(q_{0}^{\prime}, \boldsymbol{q}\right)}{q_{0}^{\prime}-q_{0}-i \varepsilon} .
$$

c) We have

$$
\left\langle\Psi_{2}\left|\left[Q_{(j)}(0), g_{0}(0)\right]\right| \Psi_{1}\right\rangle=\frac{1}{2 \pi} \int d q_{0} \tilde{F}_{00}\left(q_{0}, \Delta / 2\right) .
$$

As we have already shown in Section $2.2 \tilde{F}_{00}\left(q_{0}, \Delta / 2\right)$ receives contributions from the $E$ - and $\varphi$-parts of the representation Eq. (7) only.

We obtain

$$
\begin{gathered}
\tilde{F}_{00}\left(q_{0}, \Delta / 2\right)=\delta\left(q_{0}-\Delta_{0} / 2\right) i \int d^{4} u \int d s \delta\left(s-(u-\Delta / 2)^{2}\right) \\
\cdot E_{g_{0}}(u, s)+i P \frac{1}{q_{0}-\Delta_{0} / 2} \tilde{F}_{g_{0}}\left(q_{0}, \Delta / 2\right) .
\end{gathered}
$$

d) By combining Eqs. (36)-(38) we finally get

$$
\varkappa_{g_{0}}=\left\langle\Psi_{2}\left|\left[Q_{(j)}(0), g_{0}(0)\right]\right| \Psi_{1}\right\rangle .
$$

We note that the Eq. (36) between $\tilde{F}_{g_{v}}^{R}(q)$ and the naive retarded commutator only holds for $v=0$, i.e. we have

$$
\varkappa_{g_{v}} \neq\left\langle\Psi_{2}\left|\left[Q_{(j)}(0), g_{v}(0)\right]\right| \Psi_{1}\right\rangle \text { for } v \neq 0
$$

which is due to the non-covariance of $\left\langle\Psi_{2}\left|\left[Q_{(j)}(0), g_{v}(0)\right]\right| \Psi_{1}\right\rangle$. (40) becomes an equality only if $P_{(0), g_{\nu}}^{(2)}$ as given by (35) vanishes, i.e. if the ETC $\left[g_{0}, \partial^{\mu} j_{\mu}\right]$ contains no Schwinger-term.

We are now prepared to attack the problem of the double WI. According to our results given above we have the following WI's:

$$
\begin{aligned}
-i q_{-}^{\mu} \tilde{F}_{\mu \nu}^{R}(q) & =\tilde{F}_{g_{v}}^{R}(q)+\varkappa_{g_{v}}, \\
i q_{+}^{v} \tilde{F}_{\mu \nu}^{R}(q) & =\tilde{F}_{j_{\mu}}^{R}(q)-\varkappa_{j_{\mu}}-q_{+}^{v} P_{\mu \nu}(q)
\end{aligned}
$$

where we have defined $\tilde{F}_{g_{v}}^{R}$ and $\tilde{F}_{j_{\mu}}^{R}$ such that they satisfy normal WI's, i.e. the zeroth components of $x_{j_{\mu}}$ and $x_{g_{v}}$ respectively are the usual charge-current ETC matrix elements due to Statement 3. Therefore, the same situation as in Section 3.1 holds: The additional seagull term is defined by (21) and the solution is given by the content of Statement 1 . We are left with the physical interpretation of the consistency condition (23) in the PCAC-case. 
Statement 4. Condition (23) is equivalent to

$$
\left\langle\Psi_{2}\left|\left[Q_{(j)}(0), g_{0}(0)\right]\right| \Psi_{1}\right\rangle=\left\langle\Psi_{2}\left|\left[j_{0}(0), Q_{(g)}(0)\right]\right| \Psi_{1}\right\rangle .
$$

Proof. a) By multiplying (41) with $i q_{+}^{v}$, (42) with $-i q_{-}^{\mu}$ and using the WI's for $\tilde{F}_{g_{v}}^{R}$ and $\tilde{F}_{j_{\mu}}^{R}$ we obtain by equating the resulting r.h.s.

$$
\begin{gathered}
-\left\langle\Psi_{2}\left|\left[\partial^{\mu} j_{\mu}(0), Q_{(g)}(0)\right]\right| \Psi_{1}\right\rangle+i q_{+}^{v} \varkappa_{g_{v}} \\
=\left\langle\Psi_{2}\left|\left[Q_{(j)}(0), \partial^{v} g_{v}(0)\right]\right| \Psi_{1}\right\rangle+i q_{-}^{\mu} \varkappa_{j_{\mu}} \\
+i q_{-}^{\mu} q_{+}^{v} P_{\mu \nu}(q) .
\end{gathered}
$$

Now we put $q=-\Delta / 2$ in (44) and obtain

$$
\begin{gathered}
-\left\langle\Psi_{2}\left|\left[\partial^{\mu} j_{\mu}(0), Q_{(g)}(0)\right]\right| \Psi_{1}\right\rangle \\
=\left\langle\Psi_{2}\left|\left[Q_{(j)}(0), \partial^{v} g_{v}(0)\right]\right| \Psi_{1}\right\rangle-i \Delta^{\mu} \varkappa_{j_{\mu}} .
\end{gathered}
$$

Inserting now (45) in (44) we have

$$
i q_{+}^{v} x_{g_{v}}=i q_{+}^{\mu} x_{j_{\mu}}+i q_{-}^{\mu} q_{+}^{v} P_{\mu \nu}(q) .
$$

Therefore, due to the $q$-independence of $x_{g_{v}}$ and $x_{j_{\mu}}$, condition (23) is equivalent to

$$
x_{g_{v}}=x_{j_{v}} .
$$

b) Due to the fact that $x_{g_{v}}$ and $\chi_{j_{v}}$ transform like four vectors, (47) is already satisfied if we have it for the time component i.e., with (39), if we have (43).

\section{Applications}

4.1. Two-Point Functions. In the case of two-point functions the double WI's are a consequence of the single WI due to the symmetry

$$
\tilde{F}_{\mu \nu}^{R}(q)=\tilde{F}_{v \mu}^{R}(q)
$$

which arises from the fact that we have only one four vector $q$ on hand.

Furthermore, the ETC-term on the r.h.s. of the WI's for $\tilde{F}_{\mu \nu}^{R}$ vanishes due to TCP-invariance supposed the currents $g_{\mu}$ and $j_{\mu}$ behave equal under TCP [6]. Hence we have

$$
\begin{gathered}
-i q^{\mu} \tilde{F}_{\mu \nu}^{R}(q)=\tilde{F}_{g_{\nu}}^{R}(q), \\
i q^{v} \tilde{F}_{\mu \nu}^{R}(q)==\tilde{F}_{j_{\mu}}^{R}(q) .
\end{gathered}
$$

This leads in the conserved case to the form

and, therefore,

$$
\tilde{F}_{\mu \nu}^{R}(q)=\left(g_{\mu \nu} q^{2}-q_{\mu} q_{v}\right) F\left(q^{2}\right)
$$

$$
\tilde{F}_{\mu v}^{R}(0)=0 .
$$


We now concentrate on the case of two PCAC-currents. By applying the results of Section 3.2 we obtain:

a) An unsubtracted spectral representation for $\tilde{F}^{R}(q)$ (remember that this is just our definition of PCAC)

$$
\tilde{F}^{R}(q)=\frac{i}{2 \pi} \int d s \frac{\varphi(s)}{(q+i \varepsilon)^{2}-s} .
$$

b) The normal WI's for $\tilde{F}_{g_{\nu}}^{R}$ and $\tilde{F}_{j_{\mu}}^{R}$ respectively are a consequence of the PCAC-assumption already. This follows from the vanishing of $W_{j}^{v}$ and $P_{(0), g_{v}}^{(2)}$ in (33) (due to the fact that only one four vector $q$ is on hand). Furthermore, $E_{j}$ in (33) vanishes too, as the vacuum intermediate state in the commutator does not contribute (due to Lorentz covariance). Therefore, we have

$$
\tilde{F}_{g_{\mu}}^{R}(q)=-\tilde{F}_{j_{\mu}}^{R}(q)=\frac{q_{\mu}}{2 \pi} \int d s \frac{\varphi(s)}{s\left((q+i \varepsilon)^{2}-s\right)}
$$

and the WI

with

$$
-i q^{\mu} \tilde{F}_{j_{\mu}}^{R}(q)=\tilde{F}^{R}(q)+\left\langle 0\left|\left[Q_{(j)}(0), \partial^{v} g_{v}(0)\right]\right| 0\right\rangle
$$

$$
\left\langle 0\left|\left[Q_{(j)}(0), \partial^{v} g_{v}(0)\right]\right| 0\right\rangle=\frac{i}{2 \pi} \int d s \frac{\varphi(s)}{s} .
$$

In case of two equal currents (e.g. axial vector currents belonging to the same isospin multiplet) the $\sigma$-term (54) is different from zero due to the positivity property $\varphi(s) \geqq 0$. The most general $N$-fold subtracted spectral representation for $\tilde{F}_{\mu \nu}^{R}(q)$ solving (49) with (53) is given by

$$
\begin{aligned}
\tilde{F}_{\mu \nu}^{R}(q)= & \left(g_{\mu \nu} q^{2}-q_{\mu} q_{v}\right)\left[\frac { i } { 2 \pi } \int d s \left(\frac{\left(q^{2}+a^{2}\right)^{N}}{\left(s+a^{2}\right)^{N}} \frac{\varrho(s)}{s}\right.\right. \\
& \left.\left.-\frac{\varphi(s)}{s^{2}}\right) \frac{1}{(q+i \varepsilon)^{2}-s}+P_{(N-1)}\left(q^{2}\right)\right] \\
& +\frac{i}{2 \pi} g_{\mu \nu} \int d s \frac{\varphi(s)}{s\left((q+i \varepsilon)^{2}-s\right)} .
\end{aligned}
$$

With regard to one-particle approximations for two-point functions, we note that for subtracted spectral integrals only the low-energy part may be saturated by one-particle intermediate states. The high-energy part has to be treated by different methods.

4.2. Three-Point Functions. Anomalous WI's have been just discovered for the VAV-vertex in perturbation theory [10]. In the following we show how this phenomenon will be rediscovered within our general scheme. 
We consider the case that $j_{\mu}$ is the isospin current, $g_{v}$ the axial vector current and the states $\Psi_{2}$ and $\Psi_{1}$ are the vacuum and a one-particle $1^{-}$-state respectively, where the $1^{-}$-particle is coupled to the isospin current. If we describe the $1^{-}$-particle state by means of its four momentum $p$ and polarisation vector $\varepsilon$, then the coupling to a conserved current means that $\tilde{F}_{\mu \nu}^{R}(q)$ has to be gauge invariant, i.e.

$$
\left.\tilde{F}_{\mu \nu}^{R}(q)\right|_{\varepsilon=p}=0 .
$$

Suppose we start our game with a $\tilde{F}_{\mu \nu}^{R}$ which is gauge invariant and satisfies a normal WI with respect to $j_{\mu}$ (according to Chapter 2 and Chapter 3 this is always possible). Then the polynomial $q_{+}^{v} P_{\mu \nu}(q)=i q_{+}^{v} S_{\mu \nu}$ is gauge invariant and we must require that the seagull $S_{\mu \nu}$ has to be gauge invariant too. In accordance with the requirement $q_{-}^{\mu} S_{\mu \nu}(q)=0$ its most general form is given by

$$
S_{\mu v}(q)=\varepsilon_{\mu v \varrho \sigma}\left[\varepsilon^{\varrho} q_{-}^{\sigma} P_{1}(q, p)+p^{\varrho} q^{\sigma}(\varepsilon \cdot q) \cdot P_{2}(q, p)\right]
$$

where the $P_{i}$ 's are polynomials in $q$ and $p$. Without any loss of generality we may put $P_{2}$ equal to zero as the 2nd term in (57) does not contribute to $q_{+}^{v} P_{\mu v}$. The gauge invariance of $q_{+}^{v} S_{\mu \nu}$ is automatically satisfied due to (57). But $S_{\mu \nu}$ itself will be gauge invariant only if $P_{1} \equiv 0$, i.e. if $S_{\mu \nu} \equiv 0$. But this is not generally true.

It is interesting to investigate the AAV-vertex in the same framework. Then both currents are axialvector currents, and the states $\Psi_{1,2}$ are the same as above. In this case the double WI's are a consequence of the single WI due to crossing symmetry.

$$
\tilde{F}_{\mu \nu}^{R}(q)=-\tilde{F}_{v \mu}^{R}(-q)
$$

WI's for the three-point function $\left\langle 0\left|T^{*}\left(j_{\mu}(x) g_{v}(y) V_{\lambda}(z)\right)\right| 0\right\rangle$ in the presence of subtractions have been recently discussed by K. Wilson [12]. $\mathrm{He}$ considered exclusively the case that the singular behaviour of the three-point function has its origin in the bad behaviour for $x \rightarrow z, y \rightarrow z$. In contrast to that our scheme takes the behaviour for $x \rightarrow y$ with $V_{\lambda}$ on the mass shell into account. It is interesting to note that our results agree with the ones obtained by Wilson [12].

4.3. Four Point Functions. We will only consider the one case which is important for the application to $\pi N$ scattering [16]: $j_{\mu}$ and $g_{v}$ are both axialvector currents and $\Psi_{1,2}$ are one-nucleon states. Supposed the currents are PCAC currents then the results of Section 3.2 show that we may obtain normal double WI's if normal ETC's between axial charges and axialvector currents hold. 


\section{Conclusions}

It has been shown that the assumption of the existence of naive $T$-products and of ETC's between current densities is unnecessary for the validity of normal WI's. By means of a suitable definition of PCACcurrents we have reached conclusions which are in agreement with those obtained by Gross and Jackiw [8]:

1. $T^{*}$-products can always be defined such that WI's with respect to one current are valid.

2. The simultaneous validity of WI's with respect to two currents requires the following relation between ETC's of charges and currents

$$
\left\langle\Psi_{2}\left|\left[Q_{(j)}(0), g_{0}(0)\right]\right| \Psi_{1}\right\rangle=\left\langle\Psi_{2}\left|\left[j_{0}(0), Q_{(g)}(0)\right]\right| \Psi_{1}\right\rangle .
$$

We have obtained our results by means of subtracted JLD-representations for matrix elements of two-point retarded operators. Within the same framework the validity of WI's has been examined recently by Völkel [17]. But in contrast to our work he restricted the number of subtractions such that current-current ETC's are valid. Furthermore a technical assumption on the form of the JLD-representation for $\tilde{F}_{\mu \nu}(q)$ has been made. Völkel's results are in agreement with ours.

Our results show that the usual realization of current algebra in the form of WI's [2] is possible even if subtractions are necessary.

We conclude from the validity of normal WI's in the subtracted case:

a) The low energy theorems (soft pion results) [1] will not be altered, as they are an immediate consequence of normal WI's only.

b) A saturation of two-point functions by means of one-particle intermediate states [18] makes sense for unsubtracted spectral integrals only.

c) There is no correlation between the validity of normal WI's and the validity of the Bjorken-Johnson-Low (BJL) limit. In the subtracted case the BJL limit will not hold generally [19], as we may read off, e.g., from Eq. (14).

Acknowledgements. This work has been finished during the author's stay at the University of Karlsruhe. He is indebted to G. Höhler and J. Wess for the hospitality extended to him at their institutes.

\section{References}

1. Adler, S. L., Dashen, R. F.: Current algebra and application to particle physics. New York-Amsterdam: W. A. Benjamin 1968.

Renner, B.: Current algebras and their applications. New York: Pergamon Press 1968. 
2. Schnitzer, H. J.: Lectures presented at the NATO Advanced Study Institute, Erice (Trapani), Italy, July 1969.

3. Takahashi, Y.: Nuovo Cimento 6, 371 (1957).

4. Johnson, K.: Nucl. Phys. 25, 431 (1961);

Bjorken, J. D.: Phys. Rev. 148, 1467 (1966);

Schroer, B., Stichel, P.: Commun. Math. Phys. 3, 258 (1966), Phys. Rev. 162, 1394 (1967);

Brown, L. S.: Phys. Rev. 150, 1338 (1966);

Dietz, K., Kupsch, J.: Nucl. Phys. B 2, 581 (1967).

5. Stichel, P.: Springer Tracts Mod. Phys., 50, 110 (1969), Berlin, Heidelberg, New York: Springer.

6. Schroer, B., Stichel, P.: Commun. Math. Phys. 8, 327 (1968).

7. Brown, L. S.: Phys. Rev. 150, 1338 (1966);

Boulware, D. G., Brown, L. S.: Phys. Rev. 156, 1724 (1967);

Brown, S. G.: Phys. Rev. 158, 1444 (1967).

8. Gross, D. J., Jackiw, R.: CERN preprint TH. 1068, August 1969.

9. Dashen, R. F., Lee, S. Y.: Phys. Rev. 187, 2017 (1969).

10. Adler, St. L., Boulware, D. G.: Phys. Rev. 184, 1740 (1969).

11. Bardeen, W. A.: Phys. Rev. 184, 1848 (1969);

Jackiw, R., Johnson, K.: Phys. Rev. 182, 1459 (1969);

Gerstein, I., Jackiw, R.: Phys. Rev. 181, 1955 (1969).

12. Wilson, K. G.: Phys. Rev. 181, 1969 (1969).

13. Jost, R., Lehmann, H.: Nuovo Cimento 5, 1598 (1957).

14. Dyson, F. J.: Phys. Rev. 110, 1960 (1958).

15. Völkel, U., Völkel, A. H.: Commun. Math. Phys. 7, 261 (1968).

16. Höhler, G., Stichel, P.: University of Karlsruhe, preprint.

17. Völkel, A. H.: Private communication.

18. Schnitzer, H. J., Weinberg, S.: Phys. Rev. 164, 1828 (1967).

19. Polkinghorne, J. C.: DAMTP preprint, Cambridge 1970.

P. Stichel

II. Institut für Theoretische Physik

der Universität

D-2000 Hamburg 50, Luruper Chaussee 149 